TAIWANESE JOURNAL OF MATHEMATICS

Vol. 11, No. 4, pp. 1189-1201, September 2007

This paper is available online at http://www.math.nthu.edu.tw/tjm/

\title{
THE DUAL NOTION OF MULTIPLICATION MODULES
}

\author{
H. Ansari-Toroghy and F. Farshadifar
}

\begin{abstract}
Let $R$ be a ring with an identity (not necessary commutative) and let $M$ be a left $R$-module. In this paper we will introduce the concept of a comultiplication $R$-module and we will obtain some related results.
\end{abstract}

\section{INTRODUCTION}

Throughout this paper $R$ will denote a ring with an identity (not necessarily commutative) and all modules are assumed to be left $R$-modules. Further " $\subset$ " will denote the strict inclusion and $\mathbb{Z}$ denote the ring of integers. Let $M$ be a left $R$ module and let $S:=\operatorname{End}_{R}(M)$ be the endomorphism ring of $M$. Then $M$ has a structure as a right $S$-module so that $M$ is an $R-S$ bimodule. If $f: M \rightarrow M$ and $g: M \rightarrow M$, then $f g: M \rightarrow M$ defined by $m(f g)=(m f) g$. Also for a submodule $N$ of $M$,

$$
I^{N}:=\{f \in S: \operatorname{Im}(f)=M f \subseteq N\}
$$

and

$$
I_{N}:=\{f \in S: N \subseteq \operatorname{Ker}(f)\}
$$

are respectively a left and a right ideal of $S$. Further a submodule $N$ of $M$ is called ([4]) an open (resp. a closed) submodule of $M$ if $N=N^{\circ}$, where $N^{\circ}=$ $\sum_{f \in I^{N}} \operatorname{Im}(f)$ (resp. $N=\bar{N}$, where $\bar{N}=\cap_{f \in I_{N}} \operatorname{Ker}(f)$ ). A left $R$-module $M$ is said to be self generated (resp. self cogenerated) if each submodule of $M$ is open (resp. closed).

Let $M$ be an $R$-module. $M$ is said to be a multiplication (resp. openly multiplication) $R$-module if for every submodule $N$ of $M$ there exists a two sided ideal $I$

Received August 3, 2005, accepted March 16, 2006.

Communicated by Shun-Jen Cheng.

2000 Mathematics Subject Classification: 13C99.

Key words and phrases: Endomorphism, Multiplication modules, Comultiplication modules, Closedly comultiplication modules. 
of $R$ such that $N=I M$ (resp. $N^{\circ}=I M$ ). Recently a large body of research has been done about the left mutiplication $R$-modules haveing right $\operatorname{End}_{R}(M)$-modules structures.

Now let $M$ be an $R$-module. The purpose of this paper is to introduce the concept of comultiplication (resp. closedly comultiplication) $R$-modules (the dual notion of multiplication or openly multiplication $R$-modules). $M$ is said to be a comultiplication (resp. closedly comultiplication) $R$-module if for every submodule $N$ of $M$ there exists a two sided ideal $I$ of $R$ such that $N=\left(0::_{M} I\right)$ (resp. $\left.\bar{N}=\left(0:_{M} I\right)\right)$. It is clear that every comultiplication $R$-module is closedly comultiplicaion. It is shown that the converse is not true in general. Also we have shown that $M$ is a comultiplication $R$-module if and only if for each submodule $N$ of $M$, $N=\left(0: M A n n_{R}(N)\right)$. Furthermore, we will obtain another characterization for comultiplication $R$-modules (see 3.10) and it is shown, among the other results, that every submodule of a comultiplication $R$-module is a comultiplication $R$-module (see 3.17) and that every cocyclic module over a commutative complete Noetherian ring is a comultiplication module (see. 3.17).

\section{Auxilarly Results}

In this section we will provide the definitions and results which is necessary in the next section.

\section{Definition 2.1.}

(a) $M$ is said to be (see [6]) a multiplication module if for any submodule $N$ of $M$ there exists a two sided ideal $I$ of $R$ such that $I M=N$.

(b) Let $N$ be a non-zero submodule of $M$. Then $N$ is said to be (see [1]) large or essential (resp. small) if for every non-zero submodule $L$ of $M, N \cap L \neq 0$ (resp. $L+N=M$ implies that $L=M$ ).

(c) $M$ is said to be (see [1]) couniform if each of its non-zero submodules is small.

(d) A submodule $K$ of $M$ is called fully invariant if $K f \subseteq K$ for every $f \in$ $\operatorname{End}_{R}(M)$.

(e) Let $R$ be a commutative ring. The non-zero submodule $N$ of $M$ is said to be (see [10]) second submodule of $M$ if for each $a \in R$ the homothety $N \stackrel{a}{\rightarrow} N$ is either surjective or zero. This implies that $A n n_{R}(M)=P$ is a prime ideal of $R$.

(f) A non-zero module $M$ over a ring $R$ is said to be (see [2]) prime if the annihilator of $M$ is the same as the annihilator of $N$ for every non-zero submodule $N$ of $M$. 
(g) A non-zero module $M$ over a ring $R$ is said to be (see [2]) coprime if the annihilator of $M$ is the same as the annihilator of $Q$ for every non-zero (left) quotient $\mathrm{Q}$ of $M$.

(h) An $R$-module $M$ is said to be distributive if the lattice of its submodule is distributive, i.e. $(X+Y) \cap Z=(X \cap Z)+(Y \cap Z)$ for any of its submodules $X, Y$ and $Z$.

(i) Let $R$ be a commutative ring. An $R$-module $L$ is said to be cocyclic (see [8] and [9]) if $L \subseteq E(R / P)$ for some maximal ideal $P$ of $R$.

Remark 2.2. (see [3]). Let $R$ be a commutative Noetherian ring and let $E$ be an injective $R$-module. Then we have $\left(0:_{E}\left(0:_{R} I\right)\right)=I E$.

Lemma 2.3. Let $R$ be a commutative ring and $M$ an $R$-module. Let $S=$ $\operatorname{End}_{R}(M)$ be a domain. Then $A n n_{R}(M)$ is a prime ideal of $R$.

Proof. Let $I$ and $J$ be ideals of the ring $R$ and $I J \subseteq A n n_{R}(M)$. Then $I J M=0$. Now assume that $J M \neq 0$ and $I M \neq 0$. Hence there exist $a \in I$ and $b \in J$ such that $a M \neq 0$ and $b M \neq 0$. Consider the homotheties $M \stackrel{f_{a}}{\rightarrow} M$ and $M \stackrel{g_{b}}{\rightarrow} M$ defined respectively by $m \mapsto a m$ and $m \mapsto b m$. Then

$$
m\left(f_{a} g_{b}\right)=\left(m f_{a}\right) g_{b}=(a m) g_{b}=b a m=0 .
$$

Hence $f_{a} g_{b}=0$. Since $S$ is a domain, $f_{a}=0$ or $g_{b}=0$. Therefore, $a M=0$ or $b M=0$. But this is a contradiction. Hence $I M=0$ or $J M=0$ so that $I \subseteq A n n_{R}(M)$ or $J \subseteq A n n_{R}(M)$.

\section{MAIN Results}

Definition 3.1. An $R$-module $M$ is said to be a comultiplication module if for any submodule $N$ of $M$ there exists a two sided ideal $I$ of $R$ such that $N=\left(0:_{M} I\right)$.

Example 3.2. Let $p$ be a prime number and consider the $\mathbb{Z}$-module $M=\mathbb{Z}\left(p^{\infty}\right)$ (we recall that $\mathbb{Z}$ is the ring of integers). Choose $N=\mathbb{Z}(1 / p+\mathbb{Z})$ and Set $I=\mathbb{Z} p^{i}, i \geq 0$. It is clear that $N=\left(0:_{M} I\right)$. Therefore, $M=\mathbb{Z}\left(p^{\infty}\right)$ as a $Z$-module is a comultiplication module.

Definition 3.3. An $R$-module $M$ is said to be a closedly comultiplication module if for any submodule $N$ of $M$ there exists a two sided ideal $I$ of $R$ such that $\bar{N}=\left(0:_{M} I\right)$.

Example 3.4 Let $M$ be a duo $R$-module (i.e. every submodule of $M$ is fully invariant). Now $I_{N}$ is a two-sided ideal of $S$ and it is easy to see that 
$\bar{N}=\left(0: M I_{N}\right)$. Hence $M$ as a right $S$-module is a closedly comultiplication $S$-module.

Remark 3.5. It is clear that every comultiplication module is a closedly comultiplication module. But the following example shows that the converse is not true.

Example 3.6. As we will show in example 3.9, $\mathbb{Z}$ as a $\mathbb{Z}$-module is not a comultiplication $\mathbb{Z}$-module. However since every non-zero endomorphism of $\mathbb{Z}$ is a monomorphism, for every $\mathbb{Z}$-submodule $N$ of $\mathbb{Z}$, we have $\bar{N}=0$ or $\bar{N}=N$. This shows that $\mathbb{Z}$ is a closedly comultiplication $\mathbb{Z}$-module.

Lemma 3.7. An $R$ module $M$ is a comultiplication module if and only if for each submodule $N$ of $M, N=\left(0: M A n n_{R}(N)\right)$.

Proof. The sufficiency is clear. Conversely, suppose that $M$ is a comultiplication module. Then there exists a two sided ideal $I$ of $R$ such that $N=\left(0:{ }_{M} I\right)$. Then we have $I \subseteq A n n_{R}(N)$ so that $\left(0:_{M} A n n_{R}(N)\right) \subseteq\left(0:_{M} I\right)=N$. This implies that $N=\left(0:_{M} A n n_{R}(N)\right)$ as desired.

Example 3.8. Let $R$ be a commutative semi-simple ring and let $I$ be an ideal of $R$. Then it is clear that $R$ is both injective and Noetherian as $R$-module. Hence by Remark 2.2, we have $\left(0: R A n n_{R}(I)\right)=I R=I$. Thus every semi-simple ring as a module over itself is a comultiplication module by 3.7 .

Example 3.9. Let $M=\mathbb{Z}$ (as a $\mathbb{Z}$-module). For a submodule $2 \mathbb{Z}$ of $\mathbb{Z}$ we have $\left(0: A n n_{\mathbb{Z}}(2 \mathbb{Z})\right)=\mathbb{Z}$. Therefore, $\mathbb{Z}$ is not a comultiplication module.

Theorem 3.10. Let $M$ be an R-module. Then the following are equivalent.

(a) $M$ is a comultiplication module.

(b) For every submodule $N$ of $M$ and each two sided ideal $C$ of $R$ with $N \subset$ $\left(0::_{M} C\right)$, there exists a two sided ideal $B$ of $R$ such that $C \subset B$ and $N=(0: M B)$.

(c) For every submodule $N$ of $M$ and each two sided ideal $C$ of $R$ with $N \subset$ $\left(0:{ }_{M} C\right)$, there exists a two sided ideal $B$ of $R$ such that $C \subset B$ and $N \subseteq(0: M B)$.

Proof. $(a) \Rightarrow(b)$. Let $N$ be a submodule of $M$ and let $C$ be a two sided ideal of $R$ such that $N \subset\left(0::_{M} C\right)$. Since $M$ is a comultiplication module, $N=\left(0:{ }_{M}\right.$ $\left.A n n_{R}(N)\right)$. We set $B=C+A n n_{R}(N)$. Since $N=\left(0:_{M} A n n_{R}(N)\right) \subset\left(0:_{M}\right.$ $C), A n n_{R}(N) \not \subset C$. Hence $C \subset B$ and we have 
$\left.\left(0:_{M} B\right)=\left(0:_{M} C+A n n_{(} R N\right)\right)=\left(0:_{M} C\right) \cap\left(0:_{M} A n n_{R}(N)\right)=N$.

The implication $(b) \Rightarrow(c)$ is obvious.

$(c) \Rightarrow(a)$. Let $N$ be a submodule of $M$ and let

$$
H=\{D: D \text { is a two sided ideal of } R \text { and } N \subset(0: M D)\}
$$

Clearly $0 \in H$. Let $\left\{B_{i}\right\}, i \in I$, be any non-empty collection of two sided ideals in $H$. By assumption, $\sum_{i \in I} B_{i} \in H$. By the Zorn's Lemma, $H$ has a maximal member $C$ so that $N \subseteq\left(0:_{M} C\right)$. Assume that $N \neq\left(0:_{M} C\right)$. Then by part (c), there exists a two sided ideal $B$ with $C \subset B$ and $N \subseteq\left(0:_{M} B\right)$. But this is a contradiction by the choice of $C$. Thus we have $N=\left(0:_{M} C\right)$. This shows that $M$ is a comultiplication $R$-module.

Theorem 3.11. Let $R$ be a commutative ring and let $M$ be a comultiplication R-module. Then

(a) $M$ is a self-cogenerated $R$-module

(b) If $N$ is a submodule of $M$ such that $A n n_{R}(N)$ is a prime ideal of $R$, then $N$ is a second submodule of $M$.

\section{Proof.}

(a) Let $N$ be submodule of a comultiplication $R$-module $M$. Then there exists an ideal $I$ of $R$ such that $N=\left(0:_{M} I\right)$. For each $a \in I$, define the map $f_{a}: M \rightarrow M$ by $m \mapsto a m$. Since $R$ is a commutative ring, $f_{a}$ is an $R$ endomorphism. It is clear that for each $a \in I, N \subseteq \operatorname{Ker}\left(f_{a}\right)$ and we have

$$
\bar{N}=\cap_{f \in I_{N}} \operatorname{Ker}(f) \subseteq \cap_{a \in I} \operatorname{Ker}\left(f_{a}\right)=N .
$$

Hence $N=\bar{N}$ as desired.

(b) Set $P=A n n_{R}(N)$. Since $M$ is a comultiplication $R$-module, $N=(0: M$ $P)$. Let $\phi_{a}: N \rightarrow N$ be the non-zero $R$-homomorphism defined by $n \mapsto a n$. Let $K=\operatorname{Im} \phi_{a}=a N$. It is clear that $0 \neq K \subseteq N$. By Theorem 3.10, there exists a two sided ideal $B$ of $R$ such that $P \subset B$ and $K=\left(0:_{M} B\right)$. It follows that $B a \subseteq A n n_{R}(N)$. Since $A n n_{R}(N)$ is a prime ideal of $R$ and $P \subset B$, we have $a \in A n n_{R}(N)$ so that $a N=0$. This is a contradiction and the proof is completed.

Corollary 3.12. Let $R$ be a commutative ring and let $M$ be an $R$-module. Then $M$ is a comultiplication module if and only if it is self cogenerated and closedly comultiplication R-module.

Proof. This is an immediate consequence of 3.11 (a) and 3.5.

Corollary 3.13. Let $R$ be a commutative ring and let $M$ be a comultiplication $R$-module. Futher let $N$ be a submodule of $M$. Then The following are equivalent. 
(a) $N$ is second submodule of $M$.

(b) $A n n_{R}(N)$ is a prime ideal of $R$.

Proof. Use 3.11 (b) and 2.1 (e).

Proposition 3.14. Let $M$ be a comultiplication R-module.

(a) Let $\left\{M_{\lambda}\right\}, \lambda \in \Lambda$, be a family of submodule of module $M$ with $\cap_{\lambda \in \Lambda} M_{\lambda}=0$. Then for every submodule $N$ of $M$, we have

$$
N=\cap_{\lambda \in \Lambda}\left(N+M_{\lambda}\right) .
$$

(b) Let $P$ be a minimal two sided ideal of $R$ such that $\left(0:_{M} P\right)=0$. Then $M$ is cyclic.

\section{Proof.}

(a) Let $N$ be a submodule of $M$. Then

$$
\begin{aligned}
N & =\left(0:_{M} \operatorname{Ann}_{R}(N)\right)=\left(\cap_{\lambda \in \Lambda} M_{\lambda}:_{M} \operatorname{Ann}_{R}(N)\right) \\
& =\cap_{\lambda \in \Lambda}\left(M_{\lambda}: M A n n_{R}(N)\right) \supseteq \cap_{\lambda \in \Lambda}\left(N+M_{\lambda}\right) \supseteq N .
\end{aligned}
$$

It follows that

$$
N=\cap_{\lambda \in \Lambda}\left(N+M_{\lambda}\right)
$$

(b) Let $0 \neq m \in M$. Since $M$ is a comultiplication $R$-module, there exists a two sided ideal $I$ of $R$ such that $R m=\left(0:_{M} I\right)$ and hence

$$
R m=\left(0:_{M} I\right)=\left(\left(0:_{M} P\right):_{M} I\right)=\left(0:_{M} P I\right) .
$$

Now since $P$ is a minimal ideal of $R$ and $0 \subseteq P I \subseteq P$, we have $P I=0$ or $P I=P$. If $P I=P$, then

$$
R m=\left(0:_{M} P I\right)=\left(0:_{M} P\right)=0 .
$$

This implies that $m=0$ which is a contradiction. Hence we have $P I=0$ so that $R m=M$ as desired.

Lemma 3.15. Let $M$ be a faithful comultiplication module over a commutative ring $R$. Then $W(M)=Z(R)$, where

$$
W(M)=\{a \in R: \text { the homothety } M \stackrel{a}{\rightarrow} M \text { is not surjective }\}
$$

(here $Z(R)$ denotes the set of zero divisors of $R$ ). 
Proof. Let $a \in W(M)$ and suppose that the homothty $M \stackrel{a}{\rightarrow} M$ defined by $m \mapsto a m$ is not surjective. Then Since $M$ is a comultiplication $R$-module, there exists a two sided ideal $I$ of $R$ such that $a M=\left(0::_{M} I\right)$. Hence we have $I a M=0$ so that $I a \subseteq A n n_{R}(M)=0$. Thus $I a=0$. It follows that $a \in Z(R)$. Conversely let $a \in Z(R)$. Then there exists $0 \neq b \in R$ such that $a b=0$. Thus we have $(a b) M=(b R)(a M)=0$. This implies that $a M \subseteq\left(0:_{M} b R\right) \neq M$ because $M$ is faithful $R$-module. Therefore, $a M \neq M$ so that $a \in W(M)$.

Lemma 3.16. Let $R$ be a ring such that the lattice of two sided ideals of $R$ is distributive and let $M$ be a comultiplication R-module such that for any two sided ideal $B$ and $C$ of $R,\left(0:_{M} B\right)+\left(0:_{M} C\right)=\left(0:_{M} B \cap C\right)$. Then $M$ is $a$ distributive module.

Proof. Let $X, Y$, and $Z$ be three submodules of $M$. Since $M$ is a comultiplication module, there exist two sided ideals $B, C$ and $D$ of $R$ such that $X=\left(0:{ }_{M} B\right)$, $Y=\left(0:_{M} C\right)$ and $Z=\left(0:_{M} D\right)$. Then

$$
\begin{aligned}
& (X+Y) \cap Z=\left(\left(0:_{M} B\right)+\left(0:_{M} C\right)\right) \cap\left(0:_{M} D\right)=\left(0:_{M} B \cap C\right) \cap\left(0:_{M} D\right) \\
& =\left(0:_{M}(B \cap C)+D\right)=\left(0:_{M}(B+D) \cap(C+D)\right)=\left(0:_{M} B+D\right)+\left(0:_{M} C+D\right) \\
& =\left(\left(0:_{M} B\right) \cap\left(0:_{M} D\right)\right)+\left(\left(0:_{M} C\right) \cap\left(0:_{M} D\right)\right)=(X \cap Z)+(Y \cap Z) .
\end{aligned}
$$

Theorem 3.17. Let $M$ be a comultiplication R-module. Then the following assertions hold.

(a) Every submodule of $M$ is fully invariant.

(b) If $R$ is a commutative ring, then $\operatorname{End}_{R}(M)$ is a commutative ring.

(c) If $M$ is faithful, then $M$ is divisible.

(d) Every submodule of $M$ is a comultiplication module.

(e) If $R$ is a complete Noetherian local ring, then every cocyclic $R$-module is a comultiplication R-module.

\section{Proof.}

(a) Let $N$ be a submodule of a comultiplication $R$-module $M$. Then there exists a two sided ideal $I$ of $R$ such that $N=(0: M I)$. Suppose that $f: M \rightarrow M$ be an endomorphism. Since $I N=0, I \subseteq A n n_{R}(N f)$ so that

$$
\left(0:{ }_{M} A n n_{R}(N f)\right) \subseteq\left(0:_{M} I\right)=N .
$$

This implies that $N f \subseteq N$. 
(b) Let $f$ and $g$ be two endomorphisms of $M$ and let $m \in M$. Then we have $m f \in(R m) f$ and $m g \in(R m) g$. But by part (a), $R m(f) \subseteq R m$ and $R m(g) \subseteq R m$. Thus, $m f, m g \in R m$. So there exist elements $a, b \in R$ such that $m f=a m$ and $m g=b m$. Hence we have

$$
\begin{aligned}
m(f g-g f) & =m f(g)-m g(f)=a m(g)-b m(f) \\
& =b a m-a b m=a b m-a b m=0 .
\end{aligned}
$$

It follows that $\operatorname{End}_{R}(M)$ is a commutative ring.

(c) Let $c$ be a regular element. Then since $M$ is a comultiplication $R$-module, there exists a two sided ideal $I$ of $R$ such that $c M=\left(0:_{M} I\right)$. Since $M$ is a faithful $R$-module, we have $I c M=0$ so that $I c=0$. This implies that $I=0$ because $c$ is a regular element. Therefore, $c M=M$.

(d) Let $M$ be a comultiplication $R$ - module and let $N$ be a submodule of $M$. Let $K$ be a submodule of $N$. Then there exists a two sided ideal $I$ of $R$ such that $K=\left(0:_{M} I\right)$. But we have $K=\left(0:_{M} I\right)=\left(0:_{N} I\right)$. Therefore, $N$ is a comultiplication module.

(e) Let $P$ be the unique maximal ideal of $R$. Since every cocyclic $R$-module is a submodule of $E_{R}(R / P)$, by using part (d), it is enough to prove that $E_{R}(R / P)$ is a comultiplication $R$-module. Now by using 3.7, it is enough to prove that for every submodule $L$ of $\left.E_{R}(R / P), L=\left(0:_{E_{R}(R / P}\right) A n n_{R}(L)\right)$. To see this, set $\bar{R}=R / A n n_{R}(L), \bar{P}=P / A n n_{R}(L), \bar{E}=E_{\bar{R}} E(\bar{R} / \bar{P})$, and $\bar{H}=\left(0::_{E_{R}(R / P)} A n n_{R}(L)\right)$. Then $\bar{H}$ has a structure as $\bar{R}$-module and as such is isomorphic to $\bar{E}$. Now, as $R$ and $\bar{R}$ module, $L \subseteq \bar{H}$ and $L$ is a faithful $\bar{R}$-module. Hence by applying $\operatorname{Hom}_{\bar{R}}(-, \bar{E})$ to the exact sequence

$$
0 \rightarrow L \rightarrow \bar{H} \rightarrow \bar{H} / L \rightarrow 0
$$

one can see, as in the proof of $[6,2.3]$, that

$$
\operatorname{Hom}_{\bar{R}}(\bar{H} / L, \bar{E})=0 .
$$

This implies that $H / L=0$ as desired.

Proposition 3.18. Let $M$ be an R-module. Then the following assertions hold.

(a) If $M$ is a comultiplication prime $R$-module, then $M$ is a simple module.

(b) If $M$ is a multiplication coprime $R$-module, then $M$ is a simple module.

(c) Let $R$ be a domain and let $M$ be a faithful multiplication and comultiplication $R$ - module. Then $M$ is simple. 


\section{Proof.}

(a) Let $N$ be a non-zero submodule of $M$. Since $M$ is a prime module, we have $A n n_{R}(N)=A n n_{R}(M)$. Thus $\left(0::_{M} A n n_{R}(N)\right)=\left(0:_{M} A n n_{R}(M)\right)$. Now by using Lemma 3.7 we have

$$
N=\left(0:_{M} A n n_{R}(N)\right)=\left(0:_{M} A n n_{R}(M)\right)=M .
$$

Therefore, $M$ is a simple module.

(b) Let $M$ be a proper submodule of $M$. Since $M$ is a coprime module, we have $A n n_{R}(M)=A n n_{R}(M / N)$. Thus $A n n_{R}(M) M=A n n_{R}(M / N) M$. But $A n n_{R}(M / N) M=N$ by [5]. Hence $M$ is a simple module.

(c) Let $N$ be a submodule of a faithful multiplication and comultiplication $R$ module $M$. Then, there exist two sided ideals $I$ and $J$ of $R$ such that $N=\left(0:_{M} J\right)$ and $N=I M$. It follows that $J N=0$ so that $J I M=0$. This implies that $J I \subseteq A n n_{R}(M)=0$. So we have $J I=0$. Since $R$ is a domain, $I=0$ or $J=0$. Therefore, $N=M$ or $N=0$.

Theorem 3.19. Let $M$ be a closedly comultiplication $R$-module and $S=$ $\operatorname{End}_{R}(M)$. Then we have the following.

(a) If $N$ is a non-zero fully invariant second submodule of $M$, then $I_{N}$ is a prime ideal of $S$.

(b) If $S$ is a domain and $N$ is a closed submodule of $M$, then $I^{N}=S$ or $I^{N}$ is a prime ideal of $S$.

Proof.

(a) Since $I d_{M} \in S$ and $I d_{M} \notin I_{N}, I_{N} \neq S$. Further since $N$ is a fully invariant submodule of $M, I_{N}$ is a two sided ideal of $S$. Now let $f S g \subseteq I_{N}$, where $f, g \in S$. Then $f g \in I_{N}$. There exist two sided ideals $I$ and $J$ of $R$ such that $\operatorname{Kerf}=\left(0:_{M} I\right)$ and $K \operatorname{erg}=\left(0:_{M} J\right)$. Now $f g \in I_{N}$ implies that $N \subseteq K \operatorname{Kerfg}$. so that $N(f g)=0$. Hence $N f \subseteq \operatorname{Kerg}=\left(0::_{M} J\right)$. It follows that $0=J(N f)$ so that

$$
J N \subseteq \operatorname{Kerf}=\left(0:_{M} I\right) .
$$

This implies that $I J N=0$ so that $I J \subseteq A n n_{R}(N)$. So we have $J \subseteq$ $A n n_{R}(N)$ or $I \subseteq A n n_{R}(N)$ because $A n n_{R}(N)$ is a prime ideal of $R$ by 2.1 (e). From this we have

$$
N \subseteq(0: M J)=\text { Kerg or } N \subseteq\left(0:_{M} I\right)=K e r f .
$$

Therefore, $f \in I_{N}$ or $g \in I_{N}$ as desired. 
(b) Let $I^{N} \neq S$. Then we show that $I^{N}$ is a prime ideal of $S$. To see this let $f S g \subseteq I^{N}$. Since $1 \in S, f g \in I^{N}$. It implies that $(M) f g \subseteq N$. Also there exist two sided ideals $I, J$, and $K$ of $R$ such that $N=\left(0:{ }_{M} I\right)$, $\operatorname{Ker}(f)=\left(0:_{M} J\right)$, and $\operatorname{Ker}(g)=\left(0:_{M} K\right)$. Hence we have

$$
M(f g) \subseteq N=\left(0:_{M} I\right) .
$$

Thus $I(M(f g))=((I M) f) g=0$. This implies that

$$
(I M) f \subseteq \operatorname{Ker}(g)=\left(0:_{M} K\right) .
$$

Hence we have $(K I M) f=0$ so that

$$
(K I M) \subseteq \operatorname{Ker}(f)=(0: M J) .
$$

It follows that $J K I \subseteq A n n_{R}(M)$. Since $S$ is a domain, $A n n_{R}(M)$ is a prime ideal of $R$ so that $I \subseteq A n n_{R}(M)$ or $J \subseteq A n n_{R}(M)$ or $K \subseteq A n n_{R}(M)$. Hence $N=M$ or $\left(0:_{M} J\right)=M$ or $\left(0:_{M} K\right)=M$. So we have $I^{N}=S$ or $\operatorname{Ker}(f)=M$ or $\operatorname{Ker}(g)=M$. Since $I^{N} \neq S$, we have $\operatorname{Ker}(f)=M$ or $\operatorname{Ker}(g)=M$. If $\operatorname{Ker}(f)=M$, then $M f=0 \subseteq N$, so $f \in I^{N}$. If $\operatorname{Ker}(g)=M$, then $M g=0 \subseteq N$, so $g \in I^{N}$. Hence $I^{N}$ is a prime ideal of $S$.

Corollary 3.20. Let $M$ be a closedly comultiplication second R-module. Then $S=\operatorname{End}_{R}(M)$ is a prime ring.

Proof. It is enough to prove that the zero ideal of $S$ is a prime ideal. But by Theorem 3.19, $I_{M}=0$ is a prime ideal of $S$ as desired.

Corollary 3.21. Let $R$ be a commutative ring and $M$ be a comultiplication $R$-module. Then the following are equivalent.

(a) $S=\operatorname{End}_{R}(M)$ is a domain.

(b) $A n n_{R}(M)$ is a prime ideal of $R$.

Proof. Use 2.3, 3.20 and 3.17 (b).

Definition 3.22. Let $M$ be a comultiplication $R$-module and let $I$ is a two sided ideal of $R$. Then $\left(0:_{M} I\right)$ is said to be coidempotent if $\left(0:_{M} I\right)=\left(0:_{M} I^{2}\right)$.

Example 3.23. Let $R$ be a Noetherian ring and $I$ be an ideal of $R$. Then there exists a positive integer $h$ such that $\left(0:_{R} I^{h}\right)=\left(0:_{R} I^{h+i}\right)$ for all $i \geq 0$. Set $I^{h}=J$. Then we have $\left(0:_{R} J\right)=\left(0:_{R} J^{2}\right)$. Hence $R$ has a coidempotent $R$-submodule.

Theorem 3.24. Let $M$ be a comultiplication R-module and let $S=\operatorname{End}_{R}(M)$ be a domain. Then we have the following. 
(a) Each non-zero endomorphism of $M$ is an epimorphism.

(b) $M$ doesn't have any nontrivial open submodule.

(c) If $R$ is a commutative ring, then $M$ is a couniform $R$-module.

(d) Each closed maximal submodule of $M$ is coidempotent.

\section{Proof.}

(a) Let $0 \neq f: M \rightarrow M$ be an endomorphism of $M$. Then there exist two sided ideals $I$ and $J$ of $R$ such that $M f=\left(0:_{M} I\right)$ and $\operatorname{Ker}(f)=\left(0:_{M} J\right)$. So we have

$$
0=I\left(0:_{M} I\right)=I(M f)=(I M) f .
$$

This implies that

$$
I M \subseteq \operatorname{Ker}(f)=\left(0:_{M} J\right) .
$$

Therefore, $J I M \subseteq\left(0:_{M} J\right) J=0$ so that $J I \subseteq A n n_{R}(M)$. Since $S$ is a domain by 2.3 , we have $J \subseteq A n n_{R}(M)$ or $I \subseteq A n n_{R}(M)$. Now by using 3.7, $\operatorname{Ker}(f)=(0: M J)=M$ or $M f=(0: M I)=M$. Since $f \neq 0$, $M f=M$.

(b) Suppose that $N$ be a non-zero open submodule of $M$. Then we have

$$
N=N^{\circ}=\sum_{f \in I^{N}} \operatorname{Im}(f) .
$$

Since $0 \neq N$, there exists $0 \neq f \in S$ such that $0 \neq M f \subseteq N$. But by part (a) $M f=M$. So $N=M$.

(c) Let $N+K=M$, where, $N$ and $K$ are proper submodule of $M$. But since every comultiplication module over a commutative ring is a self cogenerated by 3.11 (a), there exist $0 \neq f, g \in S$ such that $N \subseteq \operatorname{Ker}(f)$ and $K \subseteq \operatorname{Ker}(g)$. Now we have $f g \neq 0$ because $S$ is a domain and $f, g \neq 0$. Now we have

$$
(N+K)(f g)=N(f g)+K(f g)=M(f g) .
$$

It follows that $K(f g)=M(f g)$ so that

$$
M(f g)=K(f g) \subseteq K g=0 .
$$

So we have $f g=0$. But this is a contradiction. Hence $N=M$ or $K=M$ as desired.

(d) Let $N$ be a closed maximal submodule of $M$. Then we have,

$$
M \neq N=\bar{N}=\cap_{f \in I_{N}} \operatorname{Ker}(f) .
$$

So there exists $0 \neq f \in S$ such that $N \subseteq \operatorname{Ker}(f)$. But $\operatorname{Ker}(f) \neq M$ implies that $N=\operatorname{Ker}(f)$ because $N$ is a maximal closed submodule of $M$. On the 
other hand $\operatorname{Kerf} \subseteq K \operatorname{erf} f^{2} \subseteq M$ yields that $K e r f^{2}=M$ or $K e r f^{2}=$

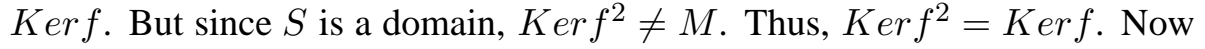
suppose that $I$ is a two sided ideal of $R$ such that $\operatorname{Kerf}=\left(0:_{M} I\right)$. Then we have $\operatorname{Kerf} f^{2}=\left(0::_{M} I^{2}\right)$ because

$$
\begin{aligned}
m \in K \operatorname{Kerf} f^{2} \Leftrightarrow & m\left(f^{2}\right)=0 \Leftrightarrow m f \in \operatorname{Kerf}=\left(0:_{M} I\right) \Leftrightarrow \\
& I(m f)=0 \Leftrightarrow I m \subseteq \operatorname{Kerf} \\
\Leftrightarrow & I^{2} m=0 \Leftrightarrow m \in\left(0:_{M} I^{2}\right) .
\end{aligned}
$$

Hence $\left(0:_{M} I\right)=\operatorname{Kerf}=\operatorname{Kerf} f^{2}=\left(0:_{M} I^{2}\right)$. This implies that $N$ is a coidempotent submodule of $M$.

Question 3.25. Let $R$ a commutative ring and let $M$ be a cocyclic $R$-module. Is $M$ a comultiplicatin $R$-module?

\section{REFERENCES}

1. W. Anderson and K. R. Fuller, Rings and categories of modules, Springer-Verlag, New York-Heidelberg-Berlin, 1914.

2. S. Annin, Associated and attached primes over noncommutative ring, $\mathrm{PhD}$. thesis, University of California at Berkeley, 2002.

3. H. Ansari-Toroghy, Associated and coassociated primes, Commun. Algebra, 26(2) (1998), 453-466.

4. Soon-Sook Bae, On submodule inducing prime ideals of endomorphism ring, East Asian Math., 16(1) (2000), 33-48

5. Z. A. El-Bast and P. F. Smith, Multiplication modules, Commun. Algebra, 16(4) (1988), 755-779.

6. Christian Lomp, Prime elements in partially ordered groupoid applied to modules and Hopfain algebra actions, Commun. Algebra, to appear.

7. R. Y. Sharp, A method for study of Artinian modules, with an application to asymptotic behaviour, in Commutative Algebra, Math Sciences Research Inst. Publ. No. 15, Springer Verlag, New York, 1986, pp. 443-464.

8. S. Yassemi, Coassociated primes, Commun. Algebra, 23 (1995), 1473-1498.

9. S. Yassemi, Coassociated primes of modules over commutative rings, Math. Scand. 80 (1997), 175-187.

10. S. Yassemi, The dual notion of prime modules, Arch. Math (Brno), 37 (2001), 273-278. 
H. Ansari-Toroghy and F. Farshadifar

Department of Mathematics,

Faculty of Science,

Guilan University,

P. O. Box 1914,

Rasht, Iran

E-mail: ansari@guilan.ac.ir 